\title{
EL PERFIL CULTURAL DE LAS GRANDES EMPRESAS EN EL ENTORNO ECONOMICO ANDALUZ. ESTADO PRESENTE Y TENDENCIAS DE FUTURO
}

\author{
Antonio LEAL MILLAN* \\ José L. GALAN GONZALEZ * \\ Ramón VALLE CABRERA * \\ Francisca MARTIN JIMENEZ* \\ M. Lucía RODRIGUEZ FELIX *
}

\section{INTRODUCCION}

El presente trabajo es el contenido de una serie de dos conferencias y mesa redonda impartidas dentro del curso de doctorado "La empresa y el empresario en Andalucía" por invitación expresa de los directores del citado curso Prof. Joaquín Guzmán Cuevas y Manuela de Paz Bañez en 1990. Dichas conferencias fueron un resumen de diversos trabajos de investigación realizados por un equipo de profesores del departamento de Organización de Empresas en el período 1987-1990.

Sobre el concepto de "cultura de las organizaciones" o cultura empresarial se está dedicando una considerable atención en la literatura reciente sobre management, pero muy pocos autores hacen referencia a investigaciones empíricas sobre el mismo. En línea a tratar de llenar este vacío, aunque solo sea modestamente, y conocer un poco más a fondo la realidad empresarial Andaluza, fueron realizadas las investigaciones que en este trabajo resumimos en dos partes diferenciadas. En la primera parte se analiza el efecto de las variables ambientales o deI entorno en la configuración de determinados perfiles culturales en las empresas de Andalucía Occidental. En la segunda parte se muestran los resultados de un

Departame'nte) de' Organizacion de Empresas. Universidad de Se'villa 
estudio prospectivo realizado con directivos y altos gerentes para conocer aquellos valores y creencias que estarán presentes en mayor medida en la cultura del management de las empresas andaluzas del futuro, y algunos de los rasgos estructurales que en opinión de los mismos dominarán el diseño de estas empresas.

¿Qué es exactamente la cultura organizacional? Casi nadie lo sabe con seguridad, y pensamos que ni tan siquiera podría darse una clara definición que sirva para siempre y que cuente con la aprobación de todos. Así, Peters y Waterman (1984, p. 75) consideran a la cultura como una representación de los valores compartidos de los miembros de una organización. Kilmann (1984, p. 11) denomina cultura "a la voluntad colectiva de los miembros" y argumenta que ello indica "lo que la corporación realmente busca o aquello con lo que realmente cuenta en orden a progresar. Schein (1983, p. 14) se refiere a la cultura como "la suma total de conocimientos y saberes compartidos o colectivos de un grupo". Schwartz y Davis (1981, p. 33) consideran a la cultura como "un modelo de creencias y expectativas compartidas por los miembros de la organización"... que producen "normas que determinan poderosamente las conductas de los individuos y grupos en la organización". Tunstall (1982, p. 1) describe a la cultura como "una constelación general de creencias, costumbres, tradiciones, sistemas de valores, normas de conducta y formas de hacer negocios que es única para cada corporación". Ouchi (1984, p. 49) expresa que "la cultura de la organización consta de una serie de símbolos, ceremonias y mitos que comunican al personal de la empresa los valores y creencias más arraigados dentro de la organización". Para Sethia y Von Glinow (1985, p. 403) la cultura organizacional podría ser definida "como el modelo compartido y relativamente duradero de valores básicos, creencias y asunciones en una organización”. Andrew Pettigrew (1979, p. 574) opina que la cultura "es un sistema de significaciones pública y colectivamente aceptadas, operacionalmente al seno de un grupo dado, en un momento dado. Este sistema de expresiones, de configuraciones y de imágenes suministra a los individuos una interpretación de su propia situación".

Podríamos continuar exponiendo las definiciones que de cultura proporcionan una gran infinidad de otros autores, no menos importantes por no citados, pero pensamos que la muestra anterior es más que suficiente para dejar de manifiesto la controversia planteada en torno al concepto de cultura organizativa.

La mayoría de estas definiciones de cultura, como hemos podido comprobar, discrepan principalmente sobre aquellos que es "compartido" por los miembros de una organización. ¿Son sus reglas, sus normas, sus creencias y expectativas, sus valores, sus filosofías, o son todas estas cosas juntas? Realmente, la discrepancia es más bien aparente que real, pues todos estos "intangibles" están inter- 
conectados, e íntimamente interrelacionados con aquello que configura poderosamente la conducta de los individuos y grupos de la organización y de esta forma la diferencia de otras organizaciones. Esta diferencia está conectada con la influencia que de hecho puede recibir la cultura de una empresa u organización cualquiera del ambiente social y económico externo más amplio en el que opera. La cultura de la organización trata de personificar aquel sistema de valores necesario para triunfar en su ambiente.

\section{LA INFLUENCIA DEL ENTORNO}

\subsection{Objetivos e hipótesis del trabajo}

Aunque cada organización o empresa es única y tiene su propia e inconfundible identidad, es posible generalizar algunos rasgos o características que suelen repetirse en muchos casos y distinguir diversas orientaciones de cultura según el entorno o medio ambiente al que se enfrentan. Aparecen entonces perfiles de rasgos culturales que caracterizan a sectores o grupos de empresas en función de determinadas adaptaciones continuas a agentes y variables exógenas que son comunes a determinadas organizaciones empresariales. Y es que, como reflejan en sus obras un gran número de autores, las culturas son "sistemas abiertos" a los entornos (Malinowski, 1977 y 1984; Parsons, 1970; Hofstede, 1980 y 1985; Hugonnier, 1982; Everett, Stening and Longton, 1982; etc...). En nuestra investigación partimos de que la cultura es real, es medible y como comprobaremos tiene una significativa relación con el entorno el sector industrial y el tipo de mercado donde se compite.

El objetivo del presente trabajo tiene pues una doble naturaleza. Por un lado, testar empíricamente la influencia que ejerce el entorno sobre la diferenciación en los rasgos culturales de las empresas: y por otro lado (y como derivación de lo anterior) conocer el estado actual de la realidad cultural de las grandes empresas de Andalucía Occidental.

Revisando la literatura sobre Organización, se sabe hoy día de la poderosa influencia que el entorno o medio ambiente ejerce sobre un conjunto de variables organizativas (véase los trabajos de Lawrence y Lorsch, 1967; Burns y Stalker, 1961; Woodward, 1965, etc.). También sabemos que un gran número de las características de las empresas de éxito tienen una fuerte naturaleza cultural (véase Peters y Waterman, 1984; Ouchi, 1984; etc.) en la medida en que la cultura es, o trata de ser, una respuesta de éxito y/o de supervivencia de las empresas frente a su entorno. 
En este sentido, y para satisfacer nuestro objetivo, hemos establecido una serie de hipótesis que tratan de sintetizar algunas de las más importantes relaciones entre el entorno y los rasgos culturales.

Estas hipótesis hacen referencia a la concepción genérica de dos tipos de entornos básicos según las condiciones de incertidumbre ambiental a las que se enfrentan las empresas, distinguiendo (según el esquema ya clásico de Lawrence y Lorsch) un entorno o ambiente dinámico de otro que puede denominarse estable. Así, se conoce que empresas del tipo de las de alta tecnología, compañías de productos de consumo, etc., operan en mercados altamente dinámicos, donde los gustos de los consumidores, los valores sociales, las fluctuaciones económicas, los desarrollos tecnológicos, etc., obligan a las mismas a frecuentes cambios en sus líneas de productos, a nuevos posicionamientos de mercado y en general a una rapídisima respuesta a las estrategias competidoras. Pero hay otras muchas empresas que no operan en mercados tan dinámicos, tales como empresas de servicios públicos, monopolios, etc., que no se mueven por cambios tan a corto plazo en las preferencias de los consumidores, que no tienen que responder tan agresivamente a la variable competencia (en parte porque no existe, véase el caso de los monopolios) y que en definitiva operan en un mercado externo más estable y con menos incertidumbre ambiental.

Estimamos que estas dos configuraciones básicas del entorno determinarán diferencias apreciables en una serie de comportamientos, valores, actitudes y en general de rasgos culturales en las organizaciones que operen dentro de una $u$ otra configuración ambiental.

Así, y como hipótesis de partida del estudio, pensamos que las empresas que se mueven en entornos estables suelen introyectar esta certidumbre o predictibilidad ambiental (unido a su bajo nivel de competencia), en su forma de actuar y reaccionar, y en definitiva dando lugar a la aparición de culturas muy centradas en torno a valores o rasgos tales como:

a) claridad de objetivos

b) integración tanto horizontal como vertical

c) compensación a través de la remuneración

d) promoción y desarrollo de carreras

Esto origina culturas muy centradas sobre la idea de estabilidad y estructuras organizativas excesivamente burocráticas (culturas "estables" en la terminología de Ansoff, 1985) donde instrumentar un cambio suele ser lento y costoso, y tal vez la propia cultura de la organización sea la barrera más importante que habrá de vencer a la hora de evolucionar hacia otras culturas más flexibles, dinámicas $\mathrm{y}$ adaptativas. 
En contraste con lo anterior, pensamos que las empresas que compiten en mercados dinámicos, más inestables y con mayores tasas de incertidumbre, cambio y competencia, desarrollarán o pondrán un mayor énfasis en valores que le ayuden a adaptarse mejor a la turbulencia de su entorno. Así, como hipótesis más concretas, creemos que estas organizaciones empresariales polarizarán sus culturas hacia aspectos o rasgos tales como:
a) el riesgo
b) la iniciativa individual
c) la espontaneidad para airear críticas y conflictos
d) el rendimiento
e) la acción

Por lo general, estas empresas que compiten en mercados dinámicos deben ser capaces de cambiar mucho más rápidamente y tienden a crear culturas más flexibles y adecuadas a la adaptación en todas las áreas de su organización (culturas "exploratorias/creativas" en la terminología de Ansoff).

\subsection{Metodología}

Para contrastar las hipótesis anteriores se escogieron diez rasgos o aspectos culturales representativos de los valores en torno a los cuales suponemos giran las diferencias culturales de las empresas según el tipo de entorno en el que actúan. Por supuesto, estos diez rasgos no intentan abarcar la globalidad y complejidad de las culturas organizacionales, sino solamente aquellos aspectos de las mismas que estimamos más directamente relacionados con la influencia del entorno. Estos son los siguientes:

1) Orientación hacia la claridad de objetivos: El grado con que la empresa utiliza y hace énfasis en crear objetivos claros y planes que los satisfagan.

2) Orientación hacia el riesgo: El grado con que la empresa fija objetivo arriesgados y enfoca o considera sus negocios atrevidos o innovadores.

3) Nivel de interdependencia horizontal: El grado con que las unidades organizativas de la empresa son estimuladas para operar de una manera coordiriada. Muestra el nivel de cooperación e integración de las unidades.

4) Nivel de interdependencia vertical: El grado con que los empleados mantienen una comunicación clara y espontánea con la alta dirección. 
5) Enfasis en la iniciativa individual: El grado con que la empresa estimula o fomenta la libertad de acción, innovación y toma de riesgos individual. Es un indicador del énfasis en el valor individualidad o personalismo.

6) Enfasis en la libre manifestación de conflictos: El grado con que los empleados son estimulados para hacer públicos y airear sus conflictos y críticas de forma abierta y francamente.

7) Orientación hacia el rendimiento: El grado con que la empresa establece y demanda altos niveles y expectativas de rendimiento a sus empleados y los hace personalmente responsables de los resultados.

8) Orientación hacia la acción: El grado con el que se da a los empleados oportunidad de realizar o implementar sus decisiones, se enfatiza un sentimiento de urgencia en conseguir resultados y cosas terminadas y se fomenta la rapidez de acción.

9) Enfasis en la remuneración competitiva: El grado con que los empleados perciben que su empresa paga competitivamente y les paga bien, así como la creencia de que se cobra según el rendimiento.

10) Orientación hacia el desarrollo de recursos humanos: El grado con que la empresa proporciona a los empleados oportunidades de crecimiento, promoción y desarrollo dentro de la misma.

Estos rasgos culturales guardan una cierta similitud con los utilizados por George C. Gordon (1985, p. 106) en su trabajo para compañías norteamericanas. No obstante, el alcance, hipótesis y metodología del mismo difieren sustancialmente respecto a nuestra investigación.

Para medir los rasgos anteriormente enumerados, elaboramos un cuestionario de 30 ítems o preguntas, de tal forma que cada tres ítems recogieran la intensidad de uno de los diez rasgos culturales establecidos, utilizando para ello una escala ordinal ( 1 a 7 ) cuyo valor central es el número 4.

Tras la elaboración del cuestionario y previo estudio del poder discriminante de cada ítem, se efectuó el trabajo de campo en una muestra de 20 grandes empresas que operan en Andalucía Occidental (provincias de Sevilla y Huelva). El tamaño de la muestra es suficientemente representativo para el nivel de confianza elegido (98\%), el error muestral aceptado $(\mathrm{E}=0,01)$ y el tamaño de la población objetivo ( $\mathrm{N}=109$ empresas de más de 250 trabajadores).

Dentro de las empresas de la muestra, el cuestionario fue cumplimentado exclusivamente por directivos de niveles superiores en la jerarquía de las mismas, en un número que oscilaba entre 10 y 15 encuestados. Y ello porque los análisis sobre las culturas empresariales revelan que dicha cultura se determina primariamente por las actitudes, valores y comportamiento directivo de los nive- 
les superiores. El comportamiento de aquellos dirigentes que se encuentran en el escalón más alto ejerce la máxima influencia, pues como señala Rensis Likert (1986, p. 11), su comportamiento directivo se refleja, y a menudo se magnifica, en los niveles sucesivamente inferiores de la organización. Centrándonos en estos altos directivos hemos obtenido, por tanto, una visión bastante clara de algunos de los más importantes valores que recorren las empresas al ser examinadas.

GRAFICO I

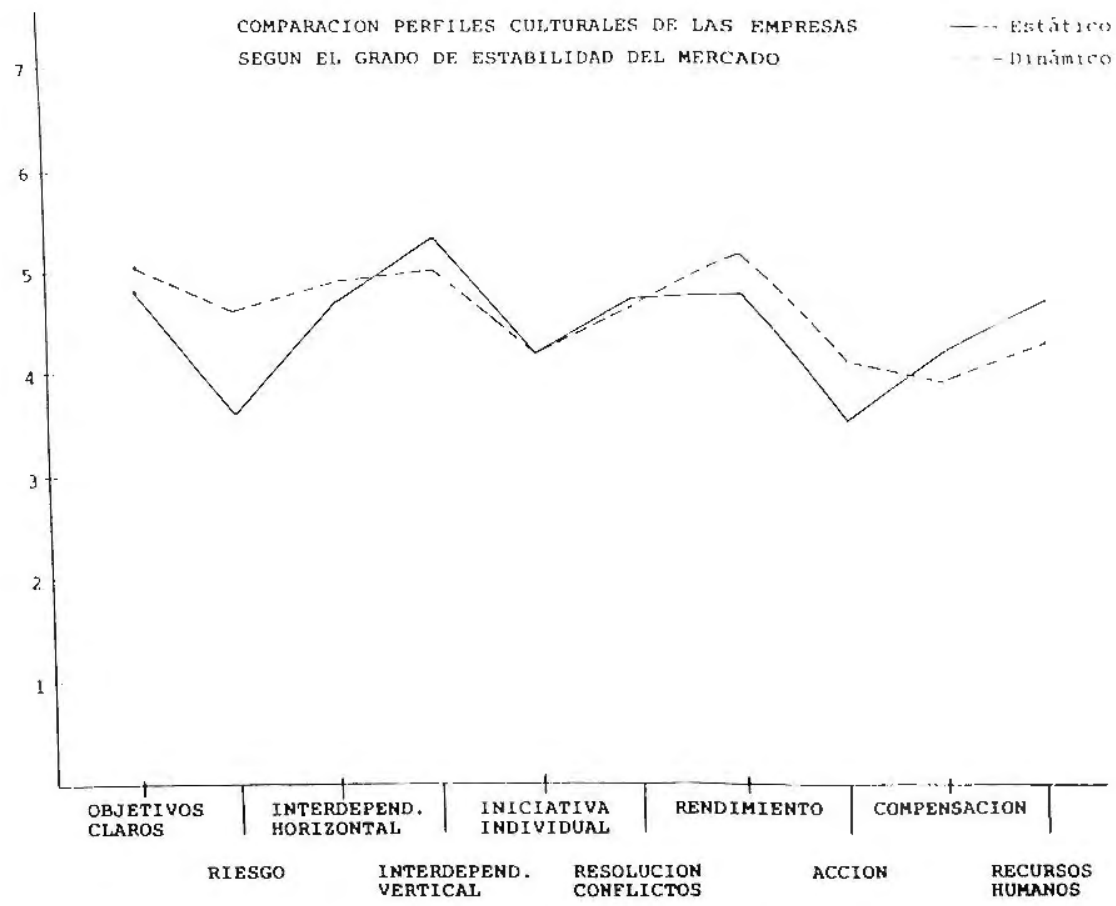

Al medir los rasgos que forman el perfil cultural de las empresas y de los sectores también hemos tenido en cuenta que los miembros de cualquier organización poseen, a grandes rasgos, dos áreas de referencia al contestar los ítems de un cuestionario. Una es la del propio grupo de trabajo o nivel del entrevistado. La segunda es la empresa en su conjunto o alguna otra entidad grande, tal como la división, la delegación territorial, etc. En nuestra investigación hemos procurado que los ítems que intentan medir un rasgo cultural en las empresas reflejen el estado conjunto de las variables correspondientes al grupo de trabajo y a la entidad organizativa global. Este estado compuesto de las variables medirá los aspectos o rasgos culturales de la empresa en general. 


\subsection{Resultados}

Los resultados del trabajo de campo se muestran en la figura 1, donde representamos gráficamente la comparación de los perfiles de rasgos culturales obtenidos tanto para las empresas que operan en entornos dinámicos como para las que lo hacen en entornos estáticos.

Una vez obtenidos los resultados de la encuesta, efectuamos además una comparación entre los perfiles de rasgos culturales elaborados en función de la pertenencia de las empresas a los distintos sectores económicos (sector industrial, comercial y de servicios). Esta comparación es la mostrada en la figura 2, la cual permite el conocimiento de una nueva dimensión con relación al entorno y, en cierta medida, satisfacer más ampliamente el segundo objetivo de este trabajo.

GRAFICO II

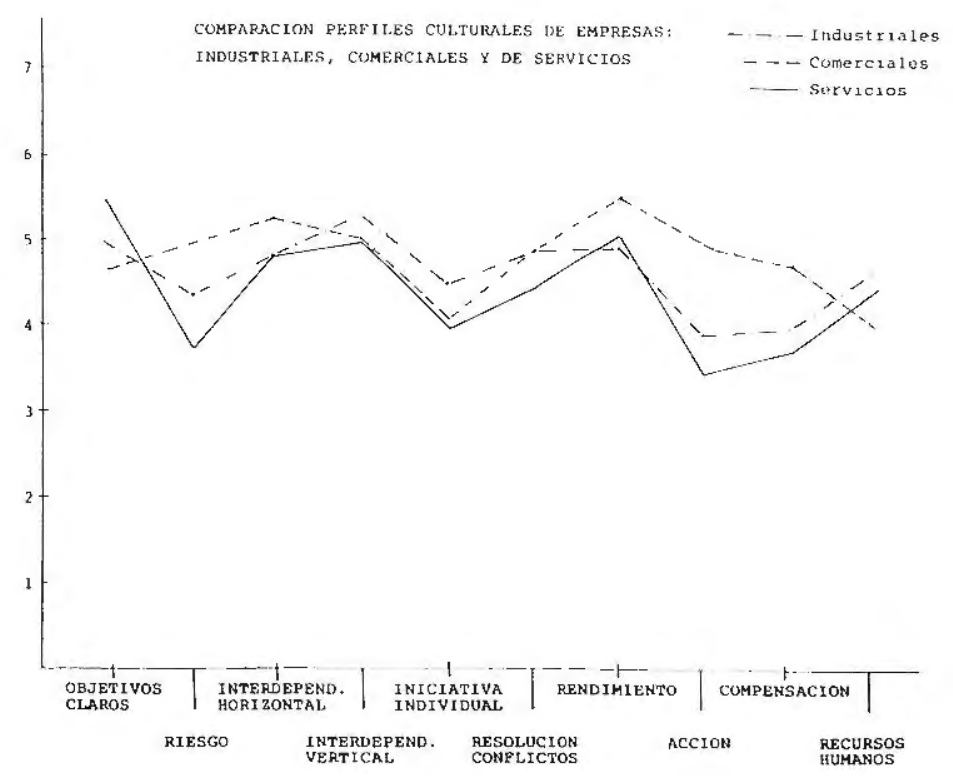

\section{LOS VALORES CULTURALES DE LA DIRECCION Y EL DISEÑO DE LAS ORGANIZACIONES EN EL FUTURO}

Como ya hemos señalado en la introducción, en esta última parte del trabajo intentamos efectuar una prospección sobre aquellos valores culturales y de diseño de las organizaciones que en opinión de profesionales y expertos, estarán presentes en mayor medida en el futuro. 
La metodología elegida para ésta prospección es la denominada como "método Delphi". Este método intenta obtener una evaluación del futuro tan precisa como sea posible en base a un desarrollo por previsión o por prospectiva. En él se utiliza como base un cuestionario que es sometido a la opinión de un grupo de expertos, en nuestro caso treinta profesionales en tareas directivas, de una forma reiterada y sucesiva. Se pretende con ello llegar a una solución o respuesta definitiva (al final del último cuestionario o fase) que de alguna forma sea la resultante de la opinión de la mayoría de los expertos.

Tras recibir los cuestionarios cumplimentados, por primera vez, se someten a un tratamiento estadístico, para lo cual se construye una distribución y se calcula la mediana y el recorrido intercuartílico para cada una de las cuestiones formuladas.

A continuación, el segundo y sucesivos cuestionarios dirigidos a los expertos incorporan los resultados estadísticos obtenidos anteriormente por el grupo, solicitándose a los mismos una nueva contestación. Puede ocurrir dos casos:

a) que la respuesta del experto se sitúe en el espacio intercuartílico, en cuyo caso las nuevas respuestas serán, presumiblemente, una repetición de las anteriores.

b) que la respuesta del experto se sitúe fuera del espacio intercuartílico. En este caso le serán solicitadas explicaciones al experto para el caso de que persista en mantener su opinión. De esta forma, sólo deben facilitar explicaciones justificativas aquellos que se sitúan fuera de la mayoría.

El cuestionario utilizado hacía referencia a dos momentos temporales futuros: años 1992 y 2000 . Los resultados obtenidos al final de la metodología (último cuestionario) han sido los que a continuación les mostramos y comentamos brevemente.

\subsection{Valores culturales futuros}

Los valores y creencias culturales en el management futuro constituyó la primera cuestión planteada (véase cuestionario en el anexo). Mediante un procedimiento consistente en entrevistar a altos directivos andaluces y solicitarles una lista de los valores y creencias que sobre el entorno, objetivos y estrategias estimaban más probables, se cruzó esta información y se seleccionaron los veinte valores culturales más repetidos. A partir de aquí, se les pidió a los expertos que formaban la muestra (distintos de los anteriores) que seleccionaran aquellos diez 
valores que consideraban estarían presentes, en mayor medida, en la cultura del futuro (años 1992 y 2000). El resultado final obtenido (último cuestionario) es el siguiente, ordenados de más o menos votados:

\section{VALORES AÑO 1992 \\ VALORES AÑO 2000}

13. Fuerte énfasis en mejorar la posición de rentabilidad de la empresa a través de disminuir los costes y nunca en base a aumentar los precios

2. Sondear y satisfacer siempre nuevas necesidades de los consumidores.

5. Fuerte énfasis en la creatividad e innovación.

11. Fuerte orientación hacia las ventas y el poder en el mercado.

1. Ofrecer siempre productos de gran calidad.

8. Logro de un fuerte compromiso del personal con los objetivos de la empresa.

10. Fuerte orientación generalizada hacia el beneficio.

15. Fuerte orientación hacia el uso de nuevas tecnologías.

16. Gran importancia del componente imagen social.

18. Fuerte orientación hacia la liquidez y generación de fondos como potencial estratégico de la cartera de negocios.
2. Sondear y satisfacer siempre nuevas necesidades de los consumidores.

5. Fuerte énfasis en la creatividad e innovación.

4. Potenciar el desarrollo integral de las personas.

8. Logro de un fuerte compromiso del personal con los objetivos de la empresa

13. Fuerte énfasis en mejorar la posición de rentabilidad de la empresa a través de disminuir los costes y nunca en base a aumentar los precios.

16. Gran importancia del componente imagen social.

1. Ofrecer siempre productos de gran calidad.

9. Fuerte compromiso de la empresa con la conservación de su entorno ecológico.

14. Fuerte orientación hacia la investigación y desarrollo de nuevas tecnologías.

17. Cooperación con otras empresas para realizar proyectos compartidos y diversificación del riesgo... 
Análogamente, se elaboró una relación de diez "valores y creencias que sobre la estructura organizativa" podrían formar parte de la cultura empresarial de los próximos años. Tras solicitar al grupo de expertos que seleccionaran los cinco más importantes, se obtuvo el siguiente resultado (de más a menos votados):

\section{VALORES AÑO 1992}

5. Gran énfasis en la transparencia y mejora de los canales de comunicación e información internos.

4. Fuerte dominio de los procesos de toma de decisiones consensuados frente a aquellos individualizados.

3. Revisión permanente de si el hombre adecuado ocupa el puesto adecuado.

1. Fuerte desarrollo de sistemas de ventas próximos al cliente.

2. Configurar unidades descentralizadas, pequeñas y de gran dinamismo.

\section{VALORES AÑO 2000}

5. Gran énfasis en la transparencia y mejora de los canales de comunicación e información internos.

3. Revisión permanente de si el hombre adecuado ocupa el puesto adecuado.

4. Fuerte dominio de los procesos de toma de decisiones consensuados frente a aquellos individualizados.

7. Fuerte predominio de la rotación de los colaboradores y del enriquecimiento del trabajo frente a la excesiva especialización.

2. Configurar unidades descentralizadas, pequeñas y de gran dinamismo.

\subsection{Rasgos estructurales en el futuro (centralización, especialización, es- tandarización y control).}

La respuesta estadística del grupo a estas dos cuestiones ha sido la que mostramos en los gráficos 3 y 4. Como puede observarse, para el año 1992 los procesos de decisión estarán dominados por la centralización, mientras que para el año 2000 se prevee una tendencia decreciente en la centralización de la toma de decisiones. 
GRAFICO III

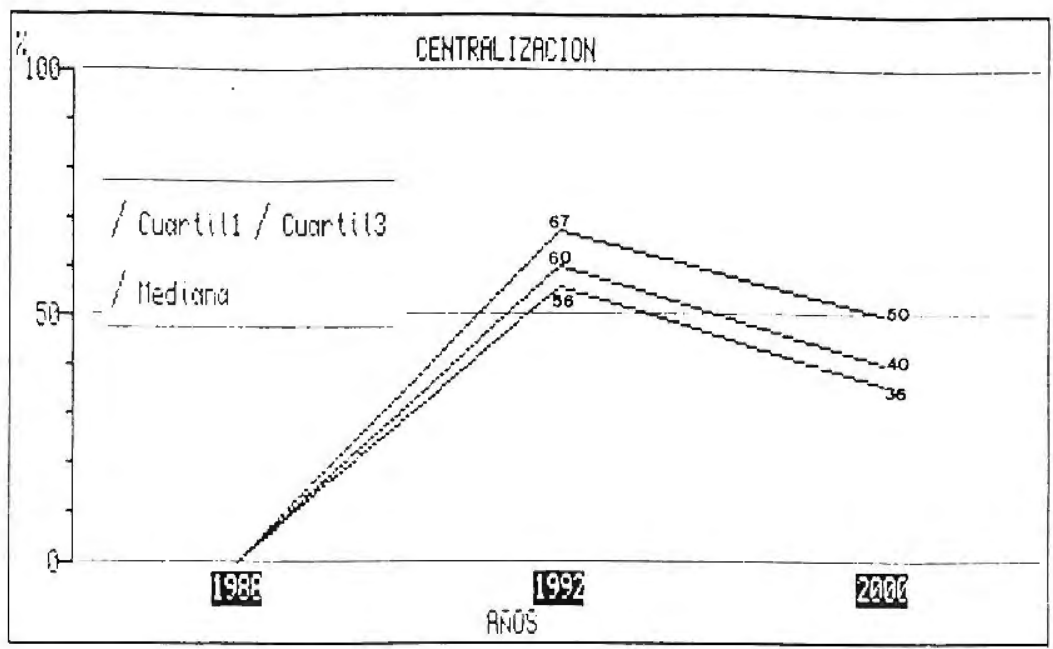

GRAFICO IV

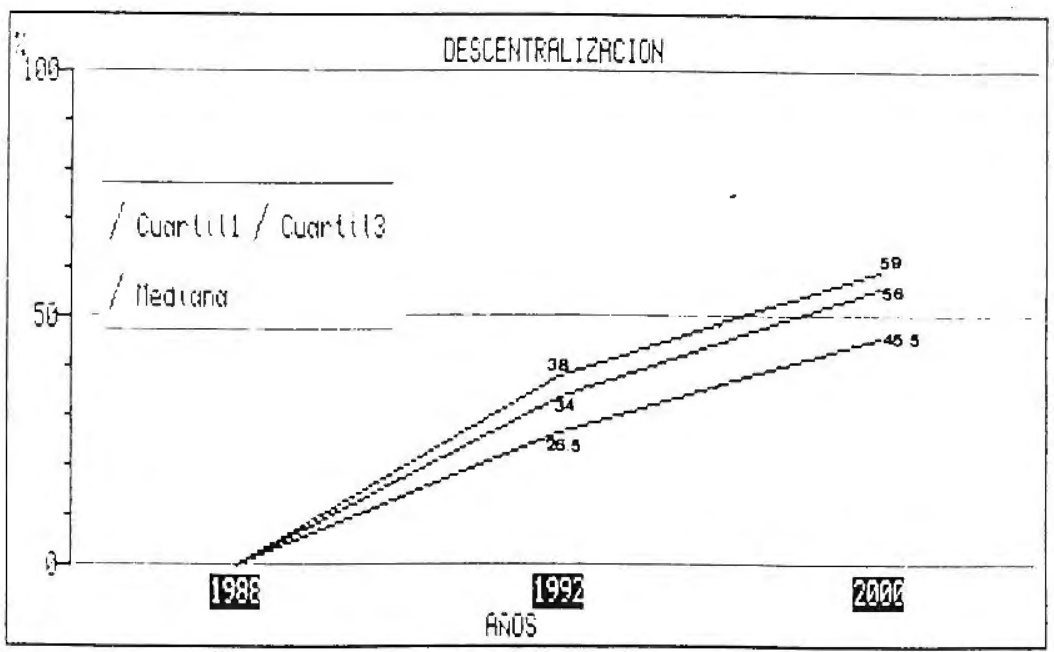

En la descentralización del proceso de toma de decisiones se ha obtenido una tendencia inversa a la anterior. Esta tendencia creciente de la descentralización es congruente dado la dicotomía del fenómeno planteado. En resumidas cuentas, la tendencia con relación al fenómeno decisor parece indicar un mayor predominio de la descentralización frente a la centralización. 
A partir de los gráficos 5 y 6 se constata que las tareas directivas, tanto para el horizonte de 1992 como del 2000, toman un valor (mediana: 3,6 y 3,7, gráfico 5) por debajo del término central de la escala de medida utilizada (de 1 a 7 ), lo cual sugiera que la especialización demandada para dichas tareas será baja.

Frente a este fenómeno de baja especialización en las tareas directivas, es de resaltar (véase gráfico 6) como para las tareas de niveles inferiores al directivo se exigirá un alto grado de especialización, según indica la tendencia de la mediana (mediana: 4,5 y 5,4 ).

GRAFICO V

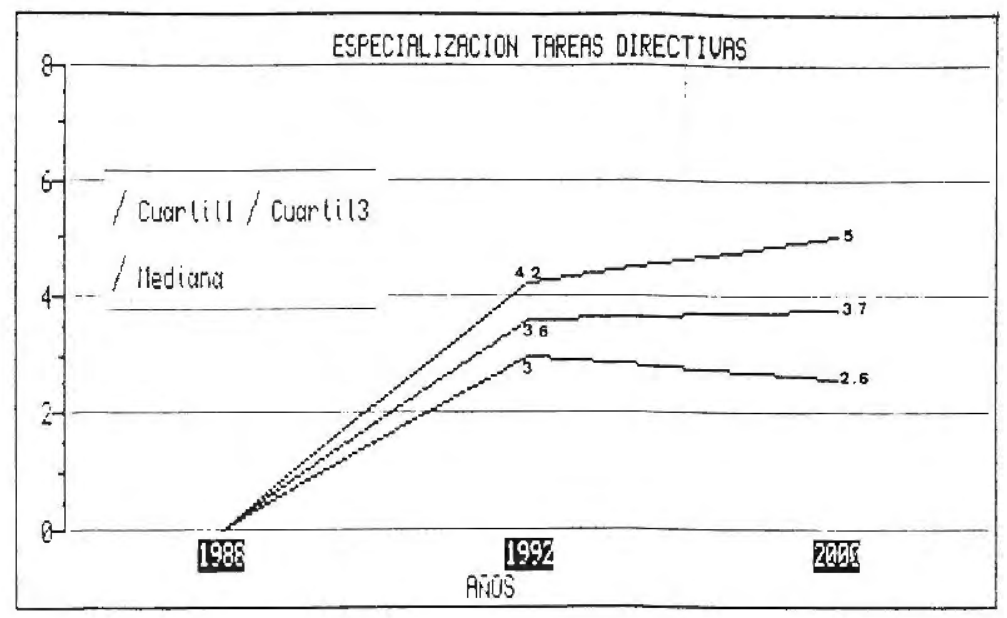

GRAFICO VI

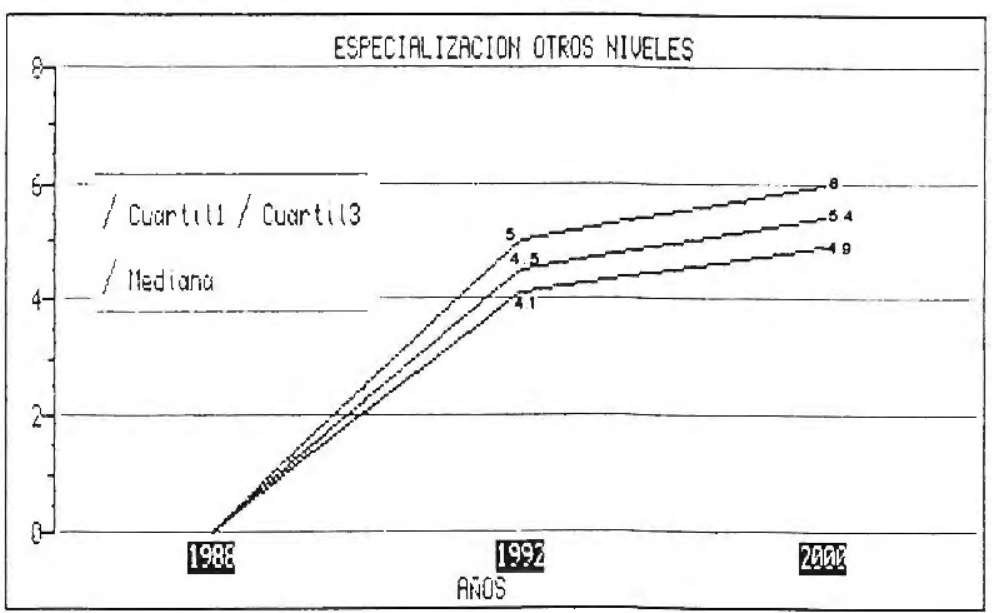


GRAFICO VII

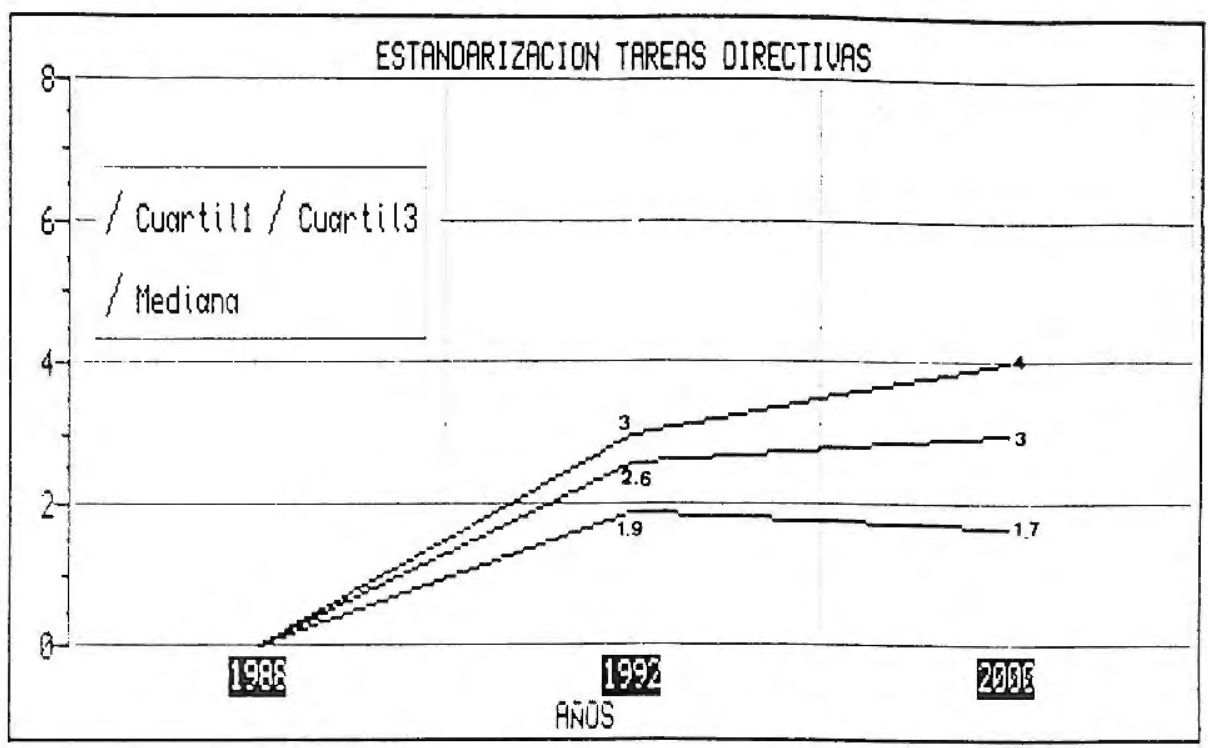

GRAFICO VIII

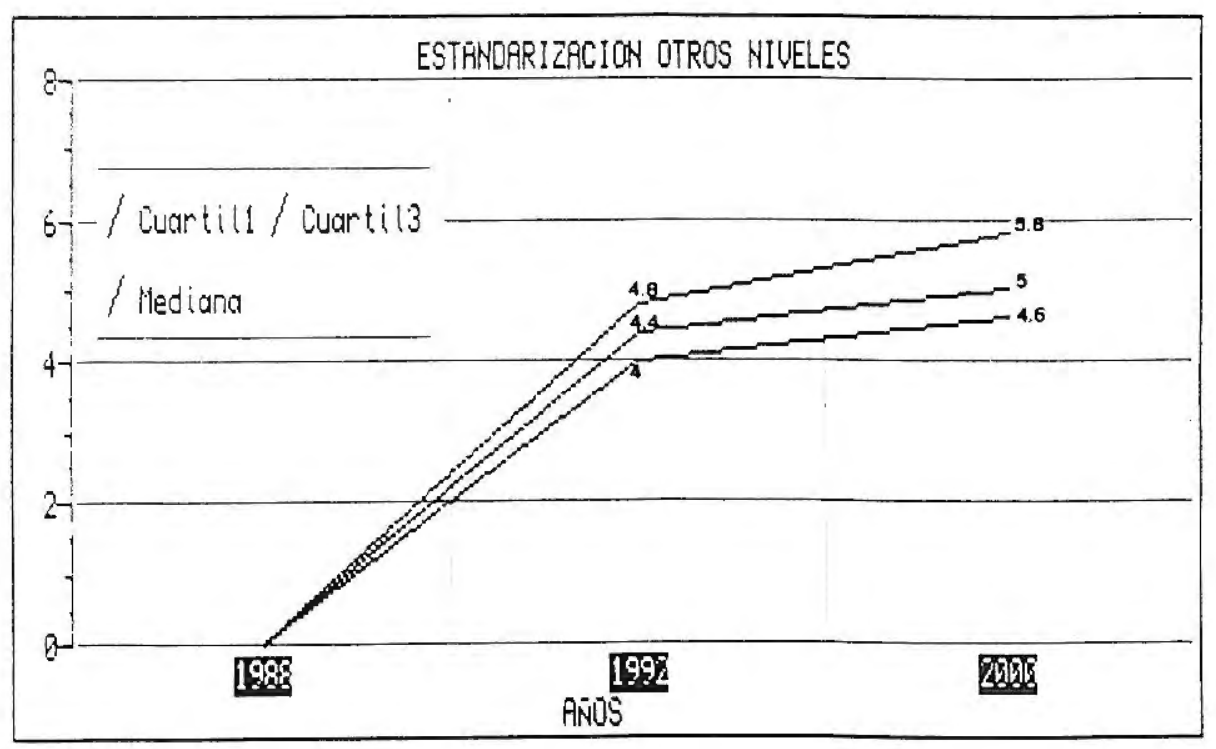


Comentario similar al de la especialización se desprende de los resultados representados en los gráficos 7 y 8 . Parece ser que en el futuro, las tareas directivas se caracterizarán por un bajo o pequeño nivel de estandarización ya que la tendencia de su mediana se mantiene por debajo del cuatro, valor central de la escala. Mientras que las tareas en otros niveles estarán dominadas por un mayor nivel de estandarización.

En opinión de los expertos consultados, el control en las organizaciones del futuro estará centrado básicamente en mecanismos y sistemas de control sobre los resultados en detrimento del control sobre los comportamientos, como bien se observa en los resultados estadísticos representados en los gráficos 9 y 10 . Merece resaltarse la alta puntuación que los expertos han concedido a control en base a los resultados, que para el año 2000 los sitúan en una banda del 5,3 al 6, 4 .

GRAFICO IX

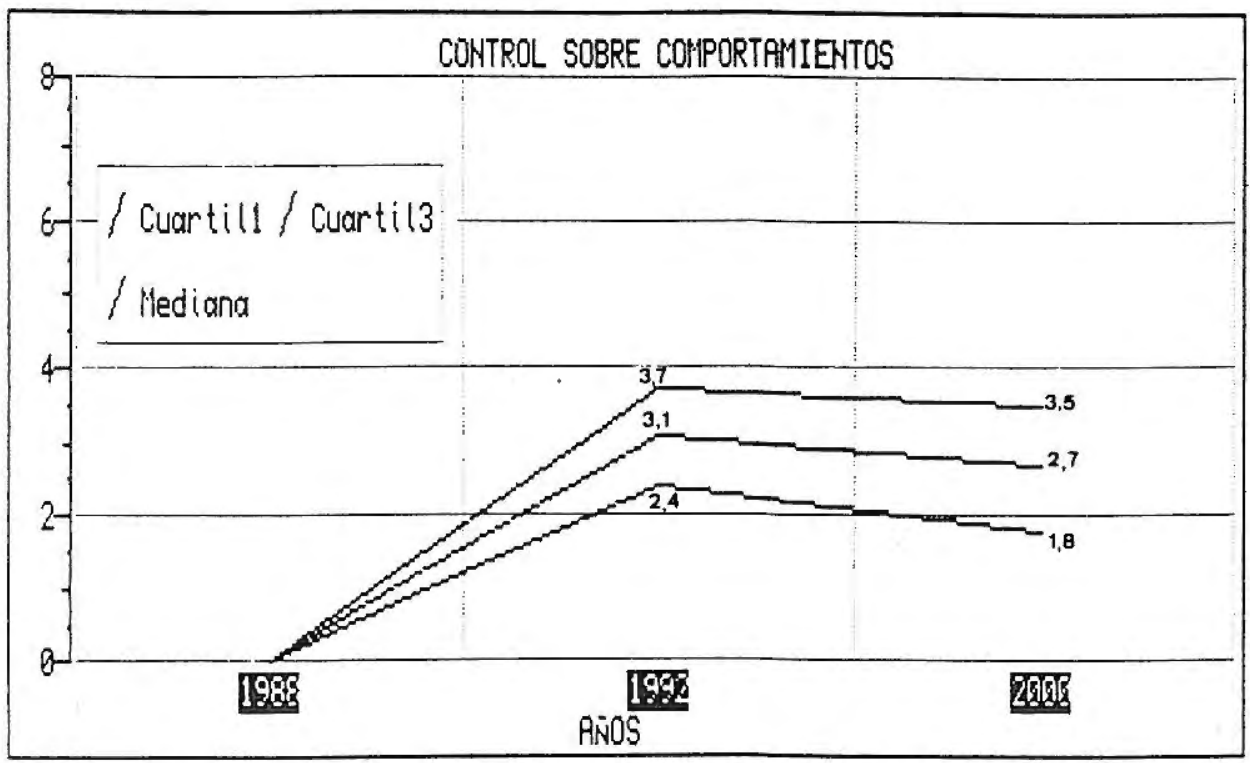


GRAFICO X

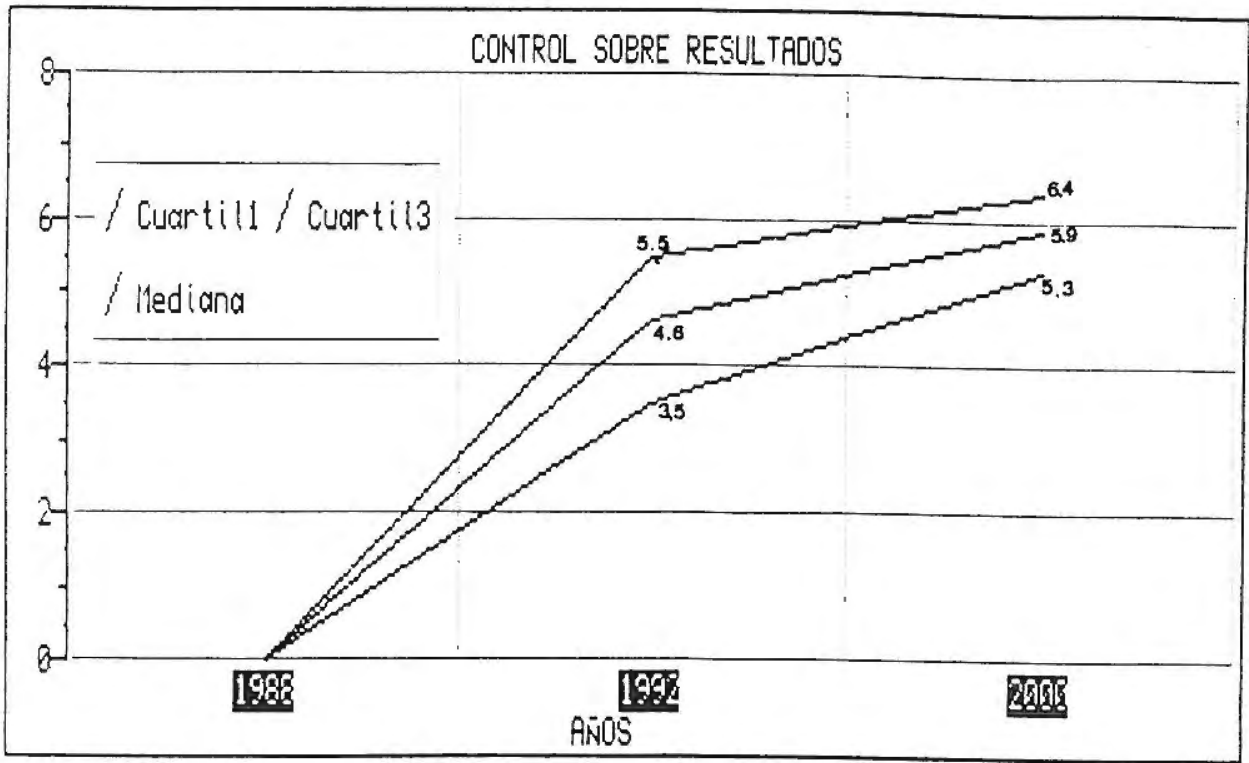

Finalmente, y como comentario general a los resultados obtenidos con esta prospección Delphi, expresar la mayor dispersión en los valores del recorrido intercuartílico del año 2000 frente a la de 1992. Esto parece lógico, habida cuenta de que conforme nos alejamos en el tiempo, la incertidumbre es cada vez mayor, lo cual se traduce en una mayor y más amplia discrepancia en cuanto a las opiniones. Este fenómeno ha tenido la única excepción en el control sobre los resultados, donde para el año 2000 se ha producido una mayor coincidencia (y por tanto menor dispersión) en las opiniones de los expertos de la muestra.

\section{CONCLUSIONES}

De los resultados del trabajo podemos deducir que la diferente configuración del entorno no provoca perfiles de rasgos culturales tan pronunciados y antagónicos como en un principio suponíamos. Esto puede obedecer a diversas razones (las cuales necesitarían un posterior análisis) entre las que pensamos se encuentran: 
a) Que en nuestra zona geográfica, las condiciones de estabilidad/dinamismo del entorno no influyan considerablemente en la acentuación de ciertos rasgos culturales en las organizaciones empresariales, tal como se podría deducir de un repaso a la literatura especializada en el tema en cuestión.

b) Que los directivos de las empresas del entorno sometido a análisis no se hayan preocupado de establecer y /o mantener culturas empresariales adaptadas a las características especiales del entorno en el que compiten, incluso generando culturas disfuncionales o contrarias a las que necesitarían desarrollar. De hecho, la cultura empresarial ha sido un recurso o activo totalmente infrautilizado $\mathrm{y} / \mathrm{o}$ gestionado convenientemente por la alta dirección de un gran número de empresas hasta fechas bastantes recientes. Incluso, hoy día, muy pocas empresas de nuestro entorno tienen un conocimiento a fondo de su propia cultura interna. Lo cual, pensamos, ha provocado la aparición de culturas espontáneas y que no siempre encarnan aquellos valores necesarios para triunfar o tener un mayor éxito en su entorno.

Así, por ejemplo, es preciso destacar que ciertas hipótesis formuladas al principio de nuestro trabajo no se han podido confirmar. Tal es el caso de los rasgos culturales: orientación hacia objetivos claros e interdependencia horizontal; que suponíamos deberían estar más acentuados en las empresas en entorno estable, y a través de los resultados hemos podido comprobar que sucede lo contrario. De igual modo, el rasgo "énfasis en la iniciativa individual" nos hace pensar que debería ser más pronunciado en las empresas que se mueven en un entorno dinámico, y esto no se ha reflejado en los datos obtenidos en la práctica.

No obstante, ciertas hipótesis establecidas sí se han confirmado (véase figura 1), como por ejemplo las referentes al grado de orientación hacia el riesgo, hacia la interdependencia vertical, hacia el rendimiento, hacia la acción y hacia el desarrollo de recursos humanos. Aunque también es cierto que de una forma no muy diferenciadora y concluyente.

En relación al segundo objetivo del trabajo, cuyos resultados se reflejan en su mayor parte en la figura 2, merece destacarse que el comportamiento de las empresas de servicios (en lo que a rasgos culturales se refiere) se ajusta bastante bien al perfil obtenido para las empresas que operan en un mercado estable. Así mismo, de la superposición de la figura 2 con la figura 1 también se puede desprender que el perfil de rasgos culturales de las empresas industriales es el que más se ajusta al obtenido para las empresas en entornos dinámicos.

Finalmente, hemos observado como las empresas comerciales del entorno andaluz, fomentan en gran medida valores culturales tales como "orientación hacia el rendimiento" y "orientación hacia la acción". Esto puede explicarse en 
términos de que las empresas comerciales que tienen una más alta rotación de pequeñas operaciones unitarias y un menor riesgo en cada una de ellas se ajustan al tipo de cultura "trabaje mucho juegue mucho" de la tipología cultural de Deal y Kennedy (1985) cuyo valor central es precisamente el de la acción.

\section{BIBLIOGRAFIA}

ANSOFF, H. I. La dirección y su actitud ante el entorno. Edic. Deusto, Bilbao, 1985.

BURNS AND STALKER. The management of innovation. Tavistock Publications, London, 1961.

DEAL, T. y KENNEDY, A. Culturas Corporativas. Fondo Educativo Interameciamo, México, 1985.

EVERETT, J.; STENING, B. y LONGTON, P. Some evidence for an international managerial culture. Joumal of Management Studies, n. 19, 1982, pp. 153-162.

GORDON, G. The relationship of corporate culture to industry sector and corporate performance. En : Kilmann, Saxton, Serpa and Associates: Gaining control of the corporate culture. Jossey-Bass, San Francisco, 1985, pp. 103-125.

HOFSTEDE, G. Culture's consequences: International differences in w'ork-related values. Sage Publications, London, 1980.

HOFSTEDE, G. The interaction between national and organizational value systems. Joumal of Management Studies. n. 22, juI. 1985, pp. 347-357.

HUGONNIER, R. Prende l'entreprise comme systeme de valeurs. Direction et Gestion, n, 6, 1982, pp. 19-30.

KILMANN R. Beyond the Quick Fix: Managing five tracks to organizational success. Jossey-Bass, San Francisco, 1984.

LAWRENCE AND LORSCH. Organization and Environment. Harvard Bussines School Press, 1967.

LIKERT, R. Nuevas formas de solucionar conflictos. Trillas, México, 1986.

MALINOWSKI, B. Los jardines del coral. Edit. Labor, Barcelona, 1977.

MALINOWSKI, B. Una teoría científica de la cultura. Edic. Sarpe, Madrid, 1984.

OUCHI, W. La teoriz Z. Edic. Orbis, S. A., Barcelona, 1984.

PARSONS, T. The system of modern societies. Prentice Hall, Englewood Cliffs, N. Jersey, 1970.

PETERS, T. y WATERMAN, R. En busca de la excelencia. Plaza y Janés, Barcelona, 1984.

PETTIGREW, A. On studying organizational cultures. Administrative Science Quarterly, december 1979, vol. 24, n. 12. pp. 570-581.

SCHEIN, E. The role of the founder in creating organizational cultures. Organizational Dynamics, $\mathrm{n} .12$ (1), 1983, pp. 13-29.

SCHWARTZ, H. y DAVIS, S. Matching corporate cultures and business strategy. Organizational Dynamics, n. $10(1), 1981$, pp. 30-48.

SETHIA, N. y VON GLINOW, M. Arriving at four cultures by managing the reward system. En: Kilmann, Saxton, Serpa and Associates: Gaining control of the corporate culture. Jossey-Bass, San Francisco, 1985, pp. 400-420.

TUNSTALL, W. Cultural transition at A.T.T.. Sloan Management Review, vol. 25, n. 1, 1983, pp. I-12.

WOODWARD, J. Industrial Organization: Theory and Practice. Oxford University Press, London, 1965. 